\title{
Variabilidade espacial da resistência do solo à penetração em plantio direto ${ }^{1}$
}

\author{
Spatial variability of the soil resistance to penetration in no tillage
}

Vanderlei Rodrigues da Silva ${ }^{2}$ José Miguel Reichert $^{3}$ Dalvan José Reinert $^{3}$

\section{RESUMO}

\begin{abstract}
O objetivo deste trabalho foi caracterizar a variabilidade espacial da resistência do solo à penetração $(R P)$ em solo sob plantio direto, identificando estados de compactação. Determinou-se a RP diretamente no campo, de forma sistemática, em três classes de solos. A primeira área experimental está localizada em Santa Maria, em um Argissolo Vermelho-Amarelo, com $120 \mathrm{~g} \mathrm{~kg}^{-1}$ de argila $\mathrm{na}$ camada de $0-20 \mathrm{~cm}$. A segunda está localizada no município de Cruz Alta em um Latossolo Vermelho distrófico, apresentando $500 \mathrm{~g} \mathrm{~kg}^{-1}$ de argila na camada de $0-20 \mathrm{~cm}$, e a terceira área experimental está localizada em Coronel Barros, em um Latossolo Vermelho distroférrico típico, com $600 \mathrm{~g} \mathrm{~kg}^{-1}$ de argila na camada de $0-20 \mathrm{~cm}$. Os valores de RP foram analisados via estatística descritiva e por geoestatística. Nos três solos, utilizou-se a técnica da krigagem para desenhar mapas de superfície, identificando três classes de RP, a qual, chamamos de estados de compactação (EC). No Argissolo, o menor EC foi considerado os valores de RP entre 0,58MPa e $0,88 \mathrm{MPa}$, o EC intermediário entre 0,89MPa e 1,18MPa e o maior EC, valores de RP entre 1,19MPa e 1,50MPa. No Latossolo Vermelho distrófico, no menor EC foram selecionados os valores de RP variando entre 1,70MPa e 2,22MPa, o EC intermediário, RP entre 2,23MPa e 2,75MPa $e$, no maior EC, valores de RP entre 2,76MPa e 3,20MPa. No Latossolo Vermelho distroférrico típico, os valores de RP no menor EC foram entre $0,20 \mathrm{MPa}$ e 1,59MPa, no EC intermediário entre 1,60MPa e 2,10MPa e no maior EC, entre 2,11MPa e 2,80MPa. Nas três áreas, a distribuição dos estados de compactação diminuiu da área de cabeceira (maior EC) para o centro da lavoura (menor EC).
\end{abstract}

Palavras-chave: compactação do solo; semivariograma, distribuição normal.

\section{ABSTRACT}

The objective of this work was to characterize the spatial variability of soil resistance to penetration $(R P)$ in no

\begin{abstract}
tillage farming, identifying the degree of soil compaction. The RP was determined directly in the field, in systematic way in three soils types. The first experimental area was in Santa Maria, in Hapludalf, with $120 \mathrm{~g}$ clay $\mathrm{kg}^{-1}$ in the layer of 0 $20 \mathrm{~cm}$. The second, located in Cruz Alta, in a Haplortox, with $500 \mathrm{~g}$ clay $\mathrm{kg}^{-1}$ in the $0-20 \mathrm{~cm}$ layer and the third experimental area located in Colonel Barros, in a Hapludox, with $600 \mathrm{~g}$ clay $\mathrm{kg}^{-1}$ in the $0-20 \mathrm{~cm}$ layer. The values of $R P$ were analyzed by descriptive statistics and for geoestatistical techniques. In the three soils, the technique of the krigeagem was used to make surface maps, identifying three classes of $R P$, the one that, we called degree compaction (EC). In Hapludalf, smallest $E C$ was considered the values of RP among $0.58 \mathrm{MPa}$ to $0.88 \mathrm{MPa}$, the middleman EC among 0.89MPa to $1.18 \mathrm{MPa}$ and largest $E C$, values of RP between 1.19MPa and 1.50MPa. In Haplortox, in the smallest EC the values of RP were selected varying among $1.70 \mathrm{MPa}$ to $2.22 \mathrm{MPa}$, the middleman $E C$, $R P$ among $2.23 \mathrm{MPa}$ to $2.75 \mathrm{MPa}$ and, in largest $E C$, values of RP among 2.76MPa to 3.20MPa. In Hapludox the values of $R P$ in smallest EC were among 0.20MPa to 1.59MPa, in the middleman EC among 1.60MPa to 2.10MPa and in largest EC, among 2.11MPa to 2.80MPa. In the three areas, the distribution of the soil degree compaction decreased of the headboard area (larger EC) for the center of the farming (smaller EC).
\end{abstract}

Key words: soil compaction, semivarigram, normal distribution.

\section{INTRODUÇÃO}

A compactação afeta a qualidade do solo e a sua avaliação é baseada na condição atual em que se encontra o solo em comparação a uma condição natural ou sem restrições ao crescimento e produtividade das culturas. A preferência em utilizar penetrógrafos para medir o estado de compactação está na praticidade e

\footnotetext{
${ }^{1}$ Parte da Tese de Doutorado do primeiro autor, apresentada ao Programa de Pós-graduação em Agronomia, Universidade Federal de Santa Maria (UFSM). Pesquisa realizada com recursos do CNPq e FAPERGS.

${ }^{2}$ Engenheiro Agrônomo, Dr., Pós-doutorando, convênio CAPES-PROBAL, Christian-Alberchts, Universitat Kiel-Alemanha. Email: silvavr@bol.com.br

${ }^{3}$ Engenheiro Agrônomo, PhD, Professor, Departamento de Solos, UFSM, Santa Maria, RS. Bolsista CNPq. E-mail: reichert@ccr.ufsm.br.dalvan@ccr.ufsm.br. Autores para correspondência.
} 
rapidez na obtenção dos resultados. Ainda, os penetrógrafos medem a resistência do solo em pequenos incrementos de profundidade, sendo úteis para avaliar camadas de maior resistência em profundidade e os valores de resistência à penetração são positivamente correlacionados com a densidade do solo. Estudando os efeitos do tráfego de máquinas, VOORHEES et al. (1978) verificaram que a RP foi mais sensível como indicador da compactação do que a densidade do solo.

A variabilidade espacial, horizontal e vertical, de diversas propriedades do solo, inclusive da RP, é dependente de fatores de formação do solo e fatores relacionados com o manejo do solo (SOUZA et al., 2001). No plantio direto, a ausência de revolvimento provoca um aumento da densidade do solo (HAKANSSON \& MEDVEDEV, 1995; KERTZMANN, 1996; KLEIN, 1998; SILVAet al., 2000a; SILVA et al., 2000b) que refletirá em maior RP.

No campo, a RP é bastante variável, sendo muito influenciada pelas condições do manejo dado ao solo e à intensidade de tráfego das máquinas agrícolas e é dependente da umidade do solo. Anualmente, o acúmulo de pressões provocadas pelo tráfego de máquinas ou pisoteio animal sobre o solo provoca locais de maior estado de compactação, principalmente, nas áreas ditas de "cabeceira" nas quais o tráfego de máquinas é mais intenso.

A geoestatística, ferramenta estatística utilizada para estudar a variabilidade espacial, possibilita a interpretação dos resultados com base na estrutura da variabilidade natural dos atributos avaliados, considerando a dependência espacial dentro do intervalo de amostragem. SOUZA et al. (1997) relatam o efeito da variabilidade espacial de outras propriedades físicas (umidade gravimétrica do solo e teor de argila) e químicas (fósforo disponível e potássio) do solo na cultura da laranjeira.

O estudo da variabilidade espacial de atributos do solo é particularmente importante em áreas sob diferentes manejos (SOUZA et al., 2001). A análise geoestatística pode indicar alternativas de manejo, não só para reduzir os efeitos da variabilidade espacial sobre a produção das culturas, como também para estimar respostas das plantas a determinadas práticas de manejo. A ação do sistema de manejo do solo e o sistema de tráfego não são uniformes em toda a área, por isso, torna-se importante analisar a distribuição espacial dos valores de RP.

UTSET \& CID (2001) encontraram comportamento distintos da variabilidade espacial da RP em condições de solo seco e de solo úmido. $\mathrm{Na}$ condição de solo seco, os autores obtiveram coeficiente de variação de $25 \%$ e ajustaram semivariograma do tipo esférico, enquanto que, quando úmido, o coeficiente de variação foi de $80 \%$ e os dados apresentaram efeito pepita puro, mostrando o efeito da variabilidade temporal da umidade do solo na variabilidade espacial da resistência à penetração. GONÇALVES et al. (1999), estudando a variabilidade temporal da umidade do solo verificaram que essa não se distribuiu de forma aleatória na área, possuindo dependência espacial bem definida no horizonte A de um Argissolo Vermelho.

O efeito de camadas de maior estado de compactação na variabilidade espacial da resistência à penetração foi verificado por ABREU (2000), num Argissolo Vermelho-Amarelo distrófico, cultivado com plantio direto. A camada de $7,5 \mathrm{~cm}$ até $17,5 \mathrm{~cm}$ possuía um maior estado de compactação e ausência de dependência espacial nessa camada, porém ocorreu dependência espacial nas profundidades de $2,5 \mathrm{~cm}$ e de $5 \mathrm{~cm}$ com ajuste de modelos exponencial e na profundidade de $30 \mathrm{~cm}$ ajustando modelo esférico.

A distribuição da compactação do solo em áreas sob plantio direto ocorre de maneira sistemática, sendo maior nas laterais da lavoura e diminuindo para o centro da lavoura. O objetivo deste estudo foi caracterizar a variabilidade espacial da resistência do solo à penetração em lavouras sob plantio direto, procurando identificar estados de compactação em função do tráfego das máquinas sobre o solo.

\section{MATERIAL E MÉTODOS}

Para este estudo, utilizaram-se três tipos de solos que ocorrem no Rio Grande do Sul. A primeira localiza-se na área experimental do Departamento de Solos, UFSM, Santa Maria, RS, latitude de $29^{\circ} 41^{\prime}$ Sul, longitude de $53^{\circ} 48^{\prime}$ Oeste e altitude de 95 metros. O solo é classificado como Argissolo Vermelho-Amarelo distrófico arênico (EMBRAPA, 1999) e o manejo da área experimental consistia em semeadura direta por aproximadamente 10 anos, cultivando soja ou milho no verão e trigo ou aveia durante o inverno.

A segunda área experimental estava localizada na propriedade do Sr. Ulfried Arns, Cruz Alta, RS, latitude $28^{\circ} 38^{\prime}$ Sul, longitude $53^{\circ} 36^{\prime}$ Oeste e altitude de 494 metros. O solo é classificado como Latossolo Vermelho distrófico (EMBRAPA, 1999), sendo que a área experimental era cultivada sob plantio direto por aproximadamente 14 anos, com culturas de soja e milho, no verão, aveia+ervilhaca e trigo, no inverno. O esquema de sucessão de culturas prevê que $50 \%$ da área cultivada no verão 
é destinada com a cultura da soja e $50 \%$ com milho e da mesma forma no inverno.

A terceira, em área localizada na propriedade do Sr. Arnaldo Yank, Coronel Barros, RS, latitude $28^{\circ}$ 22' Sul, longitude 54 ${ }^{\circ}$ 04' Oeste e altitude de 298 metros. O solo da área experimental é classificado como um Latossolo Vermelho distroférrico típico (EMBRAPA, 1999), cultivado sob plantio direto por aproximadamente 7 anos, com soja no verão e aveia ou trigo no inverno.

Foram realizadas determinações de resistência do solo à penetração (RP) em distâncias pré-definidas, utilizando penetrógrafo manual, com armazenamento eletrônico de dados (RIMIK, CP 20), para o Argissolo Vermelho-Amarelo distrófico, e um penetrógrafo de molas (SOILCONTROL ${ }^{\hat{a}}$ PENETROGRAPHER SC-60), para os Latossolos. Ambos penetrógrafos possuem cone com base de $12,83 \mathrm{~mm}$ de diâmetro e ângulo de $30^{\circ}$.

$\mathrm{Na}$ área experimental do Departamento de Solos da UFSM, Santa Maria, RS, determinou-se a RP a cada três metros no sentido norte-sul e a cada quatro metros no sentido leste-oeste, totalizando uma área de $450 \mathrm{~m}^{2}$ (Figura 1a). Nos Latossolos, determinou-se a RP em uma área de 50x30 metros a cada dois metros no sentido norte-sul e a cada cinco metros no sentido leste-oeste, totalizando 195 pontos para cada local (Figura 1b).

Os valores de RP foram analisados por estatísticas descritivas, tais como: média aritmética, mediana, moda, coeficiente de variação, desvio padrão, coeficientes de assimetria e de curtose, bem como o teste W (Kolmorgorov) (SNEDECOR \& COCHRAN, 1974).

$\mathrm{O}$ ajuste de semivariogramas foi realizado pelo software $\mathrm{GS}^{+}$(GAMMA DESIGN SOFTWARE, 1998), testando semivariogramas do tipo esférico, exponencial, linear e gaussiano. A escolha do modelo foi realizada observando-se o melhor coeficiente de correlação obtido pela técnica chamada de validação cruzada. Essa técnica consiste em retirar, individualmente, cada ponto medido da área estudada e o seu valor é estimado pelo modelo como se ele nunca existisse.

Após escolher o semivariograma de melhor ajuste à variável, realizou-se a krigagem dos dados. A krigagem estima valores entre os pontos observados considerando os parâmetros do semivariograma, sendo muito útil para confeccionar mapas de superfície com maior precisão.

Para analisar o grau de dependência espacial foi realizado uma modificação da classificação de CAMBARDELLA et al. (1994), subtraindo uma unidade do resultado da divisão entre $\mathrm{C}_{\mathrm{o}}$ e $\left(\mathrm{C}_{\mathrm{o}}+\mathrm{C}_{1}\right)$. Nesse caso, são considerados de dependência espacial forte os semivariogramas que têm um efeito pepita > 0,75 do patamar, de dependência espacial moderada quando o efeito pepita está entre 0,75 e 0,25 do patamar e de fraca dependência quando o efeito pepita é < 0,25.

\section{RESULTADOS E DISCUSSÃO}

Para o Argissolo, os valores de RP na profundidade de $5 \mathrm{~cm}, 10 \mathrm{~cm}$ e $20 \mathrm{~cm}$ apresentaram distribuição normal dos dados (Tabela 1). Nas demais profundidades, a RP não apresentou distribuição normal, conforme o teste W. No Latossolo Vermelho distrófico, a maioria das profundidades apresentou distribuição normal, com exceção das profundidades de $7,5 \mathrm{~cm}$ e $25 \mathrm{~cm}$. No Latossolo Vermelho Distroférrico de Coronel Barros, todas as profundidades apresentaram distribuição normal dos valores de RP. Esses resultados concordam com aqueles encontrados por UTSET \& CID (2001), que verificaram distribuição normal para a RP num Latossolo.

ABREU (2000), trabalhando num Argissolo Vermelho-Amarelo distrófico arênico, verificou distribuição normal para a RP nas profundidades de 5 $\mathrm{cm}, 7,5 \mathrm{~cm}, 10 \mathrm{~cm}$ e $15 \mathrm{~cm}$ e distribuição não normal para as profundidades de $2,5 \mathrm{~cm}, 12,5 \mathrm{~cm}, 17 \mathrm{~cm}$ e $30 \mathrm{~cm}$. DARIVA (2001) verificou distribuição diferente da normal nos valores de RP em 14 profundidades das 16 profundidades estudadas, em um Planossolo em Santa Maria, RS.

Em todas as profundidades, o coeficiente de variação (CV) da RP variou de $15 \%$ a $44 \%$ no Argissolo Vermelho-Amarelo distrófico, semelhantes aos encontrados por ABREU (2000) nesse mesmo tipo de solo. No Latossolo Vermelho distrófico, o CV variou entre $19 \%$ a $35 \%$ e, no Latossolo Vermelho distroférrico típico, o CV variou entre $23 \%$ a $40 \%$. Segundo WARRICK \& NIELSEN (1980), esses valores de CV são considerados médios para variáveis de solo.

$\mathrm{O}$ ajuste de semivariogramas nos valores de RP permite verificar a presença ou não de dependência espacial. No Argissolo, nas profundidades de $5 \mathrm{~cm}, 7,5 \mathrm{~cm}, 10 \mathrm{~cm}$ e $15 \mathrm{~cm}$, ajustaramse semivariogramas tipo Gaussiano (Tabela 2). Nas demais profundidades, ajustaram-se o modelo exponencial. Na superfície e até a profundidade de $12 \mathrm{~cm}$, o solo sofre os maiores efeitos do tráfego de máquinas, da atividade biológica e de ciclos de umedecimento e secagem que provocam grande variabilidade e dispersão dos valores de RP. Os semivariogramas ajustados para as profundidades de $12,5 \mathrm{~cm}$ até $25 \mathrm{~cm}$ apresentaram estacionaridade e alcance em torno de 3 metros. 
Tabela 1 - Valores de média, mediana (Med), moda (Mo), amplitude (Ampl), coeficiente de variação (CV em \%), assimetria (Ass), curtose (Curt) e teste de Kolmorow (W) para a resistência à penetração (MPa) em diferentes profundidades nas três classes de solos sob plantio direto.

\begin{tabular}{|c|c|c|c|c|c|c|c|c|}
\hline Profundidade & Média & Med & Mo & Ampl. & $\mathrm{CV}$ & Ass & Curt & W \\
\hline \multicolumn{9}{|c|}{ Argissolo Vermelho-Amarelo distrófico } \\
\hline $\mathrm{RP} 5 \mathrm{~cm}$ & 0,99 & 1,09 & 0,51 & 1,61 & 44,0 & 0,053 & $-1,035$ & $0,939^{*}$ \\
\hline $\mathrm{RP} 7,5 \mathrm{~cm}$ & 1,52 & 1,51 & 0,56 & 2,11 & 36,5 & $-0,260$ & $-0,636$ & $0,965^{\mathrm{ns}}$ \\
\hline $\mathrm{RP} 10 \mathrm{~cm}$ & 1,84 & 1,98 & 1,82 & 1,89 & 24,9 & $-1,058$ & 0,963 & $0,911^{*}$ \\
\hline $\mathrm{RP} 12,5 \mathrm{~cm}$ & 1,88 & 1,94 & 1,32 & 1,81 & 21,8 & $-0,287$ & $-0,271$ & $0,976^{\mathrm{ns}}$ \\
\hline $\mathrm{RP} 15 \mathrm{~cm}$ & 1,77 & 1,79 & 1,33 & 1,12 & 17,5 & 0,100 & $-1,024$ & $0,956^{\mathrm{ns}}$ \\
\hline $\mathrm{RP} 17,5 \mathrm{~cm}$ & 1,65 & 1,63 & 1,67 & 1,22 & 17,5 & 0,668 & 0,299 & $0,949^{\text {ns }}$ \\
\hline $\mathrm{RP} 20 \mathrm{~cm}$ & 1,62 & 1,58 & 1,18 & 1,28 & 18,5 & 0,921 & 0,896 & $0,926 *$ \\
\hline $\mathrm{RP} 22,5 \mathrm{~cm}$ & 1,51 & 1,49 & 1,27 & 1,15 & 16,9 & 0,410 & 0,167 & $0,973^{\text {ns }}$ \\
\hline $\mathrm{RP} 25 \mathrm{~cm}$ & 1,42 & 1,42 & 1,42 & 0,79 & 14,9 & $-0,104$ & $-0,642$ & $0,967^{\mathrm{ns}}$ \\
\hline \multicolumn{9}{|c|}{ Latossolo Vermelho distrófico } \\
\hline $\mathrm{RP} 5 \mathrm{~cm}$ & 2,48 & 2,40 & 2,10 & 3,92 & 31,5 & 0,528 & 0,425 & $0,971^{*}$ \\
\hline $\mathrm{RP} 7,5 \mathrm{~cm}$ & 2,48 & 2,40 & 2,10 & 3,28 & 27,8 & 0,356 & $-0,010$ & $0,968^{\text {ns }}$ \\
\hline $\mathrm{RP} 10 \mathrm{~cm}$ & 2,19 & 2,10 & 2,10 & 3,55 & 32,0 & 0,830 & 1,208 & $0,954^{*}$ \\
\hline $\mathrm{RP} 12,5 \mathrm{~cm}$ & 1,96 & 1,80 & 1,80 & 3,55 & 31,5 & 0,70 & 0,491 & $0,955^{*}$ \\
\hline $\mathrm{RP} 15 \mathrm{~cm}$ & 1,79 & 1,80 & 1,50 & 2,70 & 35,1 & 0,48 & $-0,175$ & $0,953^{*}$ \\
\hline $\mathrm{RP} 17,5 \mathrm{~cm}$ & 1,65 & 1,65 & 1,80 & 2,36 & 19,2 & 0,364 & $-0,124$ & $0,963^{*}$ \\
\hline $\mathrm{RP} 20 \mathrm{~cm}$ & 1,56 & 1,50 & 1,50 & 1,97 & 27,3 & 0,355 & $-0,149$ & $0,961 *$ \\
\hline $\mathrm{RP} 22,5 \mathrm{~cm}$ & 1,54 & 1,50 & 1,50 & 2,10 & 25,2 & 0,298 & 0,196 & $0,971^{*}$ \\
\hline $\mathrm{RP} 25 \mathrm{~cm}$ & 1,51 & 1,50 & 1,36 & 1,57 & 23,6 & 0,354 & 0,679 & $0,978^{\mathrm{ns}}$ \\
\hline \multicolumn{9}{|c|}{ Latossolo Vermelho distroférrico típico } \\
\hline $\mathrm{RP} 5 \mathrm{~cm}$ & 1,67 & 1,64 & 1,10 & 4,03 & 39,6 & 0,841 & 0,822 & $0,945^{*}$ \\
\hline $\mathrm{RP} 7,5 \mathrm{~cm}$ & 1,82 & 1,80 & 1,65 & 3,61 & 32,6 & 0,659 & 0,611 & $0,963^{*}$ \\
\hline $\mathrm{RP} 10 \mathrm{~cm}$ & 1,83 & 1,80 & 1,50 & 2,83 & 29,2 & 0,567 & 0,104 & $0,957^{*}$ \\
\hline $\mathrm{RP} 12,5 \mathrm{~cm}$ & 1,73 & 1,65 & 1,36 & 3,16 & 29,8 & 0,734 & 0,741 & $0,955^{*}$ \\
\hline $\mathrm{RP} 15 \mathrm{~cm}$ & 1,53 & 1,50 & 1,50 & 2,36 & 28,1 & 0,782 & 0,519 & $0,941^{*}$ \\
\hline $\mathrm{RP} 17,5 \mathrm{~cm}$ & 1,35 & 1,10 & 1,10 & 1,81 & 24,9 & 0,347 & $-0,145$ & $0,961^{*}$ \\
\hline $\mathrm{RP} 20 \mathrm{~cm}$ & 1,29 & 1,23 & 1,10 & 1,51 & 23,7 & 0,593 & 0,786 & $0,952 *$ \\
\hline $\mathrm{RP} 22,5 \mathrm{~cm}$ & 1,29 & 1,23 & 1,10 & 1,66 & 23,5 & 0,388 & 0,278 & $0,961^{*}$ \\
\hline $\mathrm{RP} 25 \mathrm{~cm}$ & 1,29 & 1,23 & 1,10 & 1,78 & 24,2 & 0,256 & 0,095 & $0,971^{*}$ \\
\hline
\end{tabular}

NS= não significativos em $5 \%$ de probabilidade. * significativo, apresentando distribuição normal.

No Argissolo, apenas nas profundidades de $7,5 \mathrm{~cm}$ e de $10 \mathrm{~cm}$, os modelos ajustados apresentaram moderada dependência espacial, conforme classificação adaptada de CAMBARDELLA et al. (1994). Nas demais profundidades, os modelos ajustados apresentaram forte dependência espacial, indicando bom ajuste dos semivariogramas aos dados amostrados (Tabela 2).

ABREU (2000) observou que a RP apresentou ausência total de dependência espacial na camada de maior estado de compactação, que se estendia da profundidade de $7,5 \mathrm{~cm}$ a $17,5 \mathrm{~cm}$, enquanto que, na profundidade de $2,5 \mathrm{~cm}, 5 \mathrm{~cm}$ e $30 \mathrm{~cm}$, os valores de RP apresentaram dependência espacial e ajustou-se o modelo exponencial e modelo esférico para a RP na profundidade de $30 \mathrm{~cm}$, em um Argissolo Vermelho-Amarelo.

Os semivariogramas ajustados para o Latossolo Vermelho distrófico foram do tipo exponencial para as profundidades de $5 \mathrm{~cm}, 10 \mathrm{~cm}, 17,5$ $\mathrm{cm}, 20 \mathrm{~cm}$ e $25 \mathrm{~cm}$, e do tipo Gaussiano para as profundidades de $7,5 \mathrm{~cm}, 12,5 \mathrm{~cm}$ e $15 \mathrm{~cm}$ (Tabela 2). Nas profundidades de $5 \mathrm{~cm}$ até $15 \mathrm{~cm}$, os semivariogramas apresentaram alcance entre 18,7 a 25,2 metros (Tabela 2). Nas profundidades de $17,5 \mathrm{~cm}, 20 \mathrm{~cm}$ e $25 \mathrm{~cm}$, o alcance da dependência espacial foi de aproximadamente 13,6 metros. Para esse tipo de solo e nessa condição de manejo, os modelos apresentaram moderada dependência espacial, em torno de 0,5 (Tabela 2). Apesar de apresentarem moderada dependência espacial, o coeficiente de determinação 
Tabela 2 - Estimativa dos parâmetros efeito pepita $\left(C_{0}\right)$, patamar $\left(C_{0}+C_{1}\right)$, alcance $(A)$, relação efeito pepita/patamar $\left(1-\left(C_{0} / C_{0}+C_{1}\right)\right)$ e modelo ajustados aos semivariogramas, para as três classes de solos.

\begin{tabular}{|c|c|c|c|c|c|c|c|}
\hline Variável & $\mathrm{C}_{\mathrm{o}}$ & $\mathrm{C}_{\mathrm{o}}+\mathrm{C}_{1}$ & $\mathrm{~A}(\mathrm{~m})$ & $1-\left(\mathrm{C}_{\mathrm{o}} / \mathrm{C}_{\mathrm{o}}+\mathrm{C}_{1}\right)$ & $r^{2}$ & $\mathrm{VC}^{1}$ & Modelo \\
\hline \multicolumn{8}{|c|}{ Argissolo Vermelho-Amarelo distrófico } \\
\hline $\mathrm{RP} 5 \mathrm{~cm}$ & 0,137 & 0,843 & 67,6 & 0,837 & 0,959 & 0,408 & Gaussiano \\
\hline $\mathrm{RP} 7,5 \mathrm{~cm}$ & 0,219 & 0,746 & 45,4 & 0,706 & 0,939 & 0,516 & Gaussiano \\
\hline $\mathrm{RP} 10 \mathrm{~cm}$ & 0,166 & 0,457 & 52,4 & 0,636 & 0,696 & 0,448 & Gaussiano \\
\hline $\mathrm{RP} 12,5 \mathrm{~cm}$ & 0,021 & 0,194 & 2,43 & 0,891 & 0,449 & 0,412 & Exponencial \\
\hline $\mathrm{RP} 15 \mathrm{~cm}$ & 0,021 & 0,104 & 3,98 & 0,794 & 0,144 & 0,281 & Gaussiano \\
\hline $\mathrm{RP} 17,5 \mathrm{~cm}$ & 0,007 & 0,080 & 2,21 & 0,917 & 0,215 & 0,274 & Exponencial \\
\hline $\mathrm{RP} 20 \mathrm{~cm}$ & 0,009 & 0,089 & 2,86 & 0,898 & 0,619 & 0,276 & Exponencial \\
\hline $\mathrm{RP} 25 \mathrm{~cm}$ & 0,005 & 0,044 & 2,60 & 0,896 & 0,674 & 0,189 & Exponencial \\
\hline \multicolumn{8}{|c|}{ Latossolo Vermelho distrófico } \\
\hline $\mathrm{RP} 5 \mathrm{~cm}$ & 0,351 & 0,732 & 23,8 & 0,520 & 0,937 & 0,686 & Exponencial \\
\hline $\mathrm{RP} 7,5 \mathrm{~cm}$ & 0,284 & 0,568 & 20,6 & 0,501 & 0,997 & 0,557 & Gaussiano \\
\hline $\mathrm{RP} 10 \mathrm{~cm}$ & 0,198 & 0,599 & 18,7 & 0,669 & 0,989 & 0,552 & Exponencial \\
\hline $\mathrm{RP} 12,5 \mathrm{~cm}$ & 0,250 & 0,501 & 23,3 & 0,501 & 0,971 & 0,503 & Gaussiano \\
\hline $\mathrm{RP} 15 \mathrm{~cm}$ & 0,218 & 0,447 & 25,2 & 0,513 & 0,970 & 0,460 & Gaussiano \\
\hline $\mathrm{RP} 17,5 \mathrm{~cm}$ & 0,115 & 0,242 & 13,8 & 0,525 & 0,937 & 0,391 & Exponencial \\
\hline $\mathrm{RP} 20 \mathrm{~cm}$ & 0,101 & 0,203 & 13,6 & 0,502 & 0,786 & 0,366 & Exponencial \\
\hline $\mathrm{RP} 25 \mathrm{~cm}$ & 0,065 & 0,131 & 13,6 & 0,504 & 0,897 & 0,313 & Exponencial \\
\hline \multicolumn{8}{|c|}{ Latossolo Vermelho Distroférrico típico } \\
\hline $\mathrm{RP} 5 \mathrm{~cm}$ & 0,119 & 0,545 & 12,9 & 0,782 & 0,920 & 0,505 & Exponencial \\
\hline $\mathrm{RP} 7,5 \mathrm{~cm}$ & 0,199 & 0,423 & 17,0 & 0,528 & 0,932 & 0,482 & Gaussiano \\
\hline $\mathrm{RP} 10 \mathrm{~cm}$ & 0,169 & 0,341 & 17,2 & 0,501 & 0,901 & 0,480 & Gaussiano \\
\hline $\mathrm{RP} 12,5 \mathrm{~cm}$ & 0,196 & $---^{2}$ & ---- & 0,442 & 0,891 & 0,489 & Linear \\
\hline $\mathrm{RP} 15 \mathrm{~cm}$ & 0,138 & ---- & ---- & 0,425 & 0,961 & 0,412 & Linear \\
\hline $\mathrm{RP} 17,5 \mathrm{~cm}$ & 0,089 & 0,179 & 41,1 & 0,503 & 0,966 & 0,322 & Gaussiano \\
\hline $\mathrm{RP} 20 \mathrm{~cm}$ & 0,062 & ---- & ---- & 0,423 & 0,883 & 0,275 & Linear \\
\hline $\mathrm{RP} 25 \mathrm{~cm}$ & 0,086 & ---- & ---- & 0,215 & 0,841 & 0,311 & Linear \\
\hline
\end{tabular}

${ }^{1}$-Validação Cruzada. ${ }^{2}$ não existe para esse tipo de semivariograma ajustado.

$\left(r^{2}\right)$ da relação entre a semivariância e o modelo ajustado foi superior a 0,80 (exceção na profundidade de $20 \mathrm{~cm}$ que o $r^{2}$ foi de 0,78 ).

No Latossolo Vermelho distroférrico, os semivariogramas ajustados foram do tipo exponencial na profundidade de $5 \mathrm{~cm}$, tipo gaussiano nas profundidades de $7,5 \mathrm{~cm}, 10 \mathrm{~cm}$ e $17,5 \mathrm{~cm}$ (Tabela 2). Nas profundidades de $12,5 \mathrm{~cm}, 15 \mathrm{~cm}, 20 \mathrm{~cm}$ e $25 \mathrm{~cm}$, os dados de RP apresentaram um comportamento crescente com a distância, ajustando-se então, semivariograma do tipo linear. Na profundidade de $5 \mathrm{~cm}$, o alcance da dependência espacial foi de aproximadamente 13 metros, já nas profundidades de $5 \mathrm{~cm}, 7,5 \mathrm{~cm}$ e $10 \mathrm{~cm}$, os valores de alcance ficaram em torno de 17 metros. $\mathrm{O}$ modelo linear caracteriza-se por não apresentar alcance. Na profundidade de $5 \mathrm{~cm}$, o semivariograma apresentou forte dependência espacial (Tabela 2). Na profundidade de $25 \mathrm{~cm}$, o semivariograma apresentou fraca dependência espacial, enquanto que, as demais profundidades apresentaram moderada dependência espacial.

SOUZA et al. (2001), analisando a variabilidade espacial de atributos físicos de um Latossolo Vermelho distrófico sob semeadura direta em Selvíria (MS), ajustaram semivariograma do tipo esférico para a RP na profundidade de $0-5 \mathrm{~cm}$, com alcance de $41,6 \mathrm{~m}$ e modelo exponencial na profundidade de $15-20 \mathrm{~cm}$, com alcance de $58,8 \mathrm{~m}$, ambos apresentando moderada dependência espacial.

Para visualizar a distribuição dos valores de RP nos locais estudados, realizou-se a krigagem dos dados e gerando mapas de superfície. No Argissolo, o maior estado de compactação (EC) estendeu-se da entrada da lavoura, com RP variando de 1,19 até 1,50MPa, até aproximadamente os 12 metros iniciais (Figura 1a). Na seqüência, o estado de compactação intermediário foi considerado a RP variando de 0,89 a $1,18 \mathrm{MPa}$ e estendeu-se dos 12 


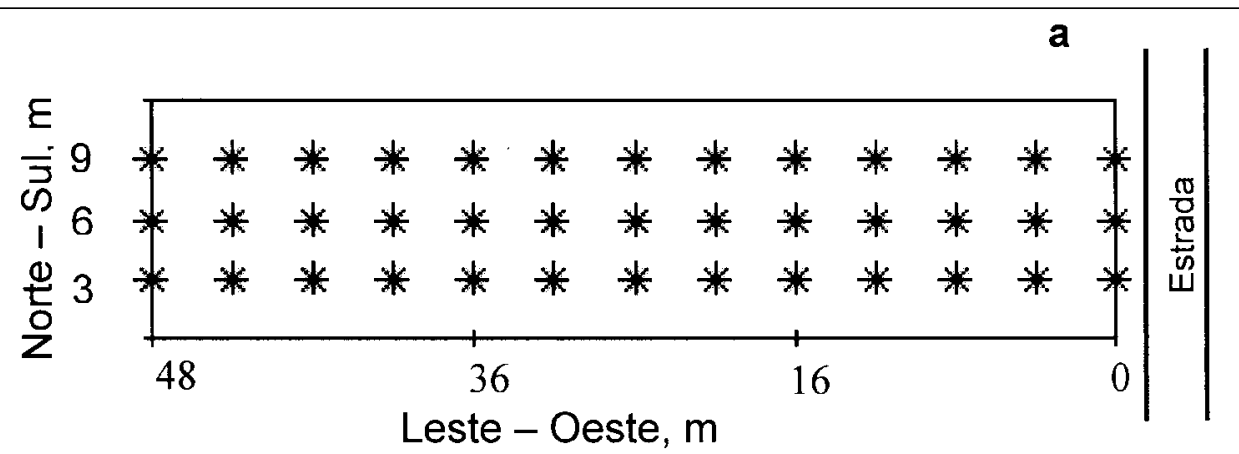

b

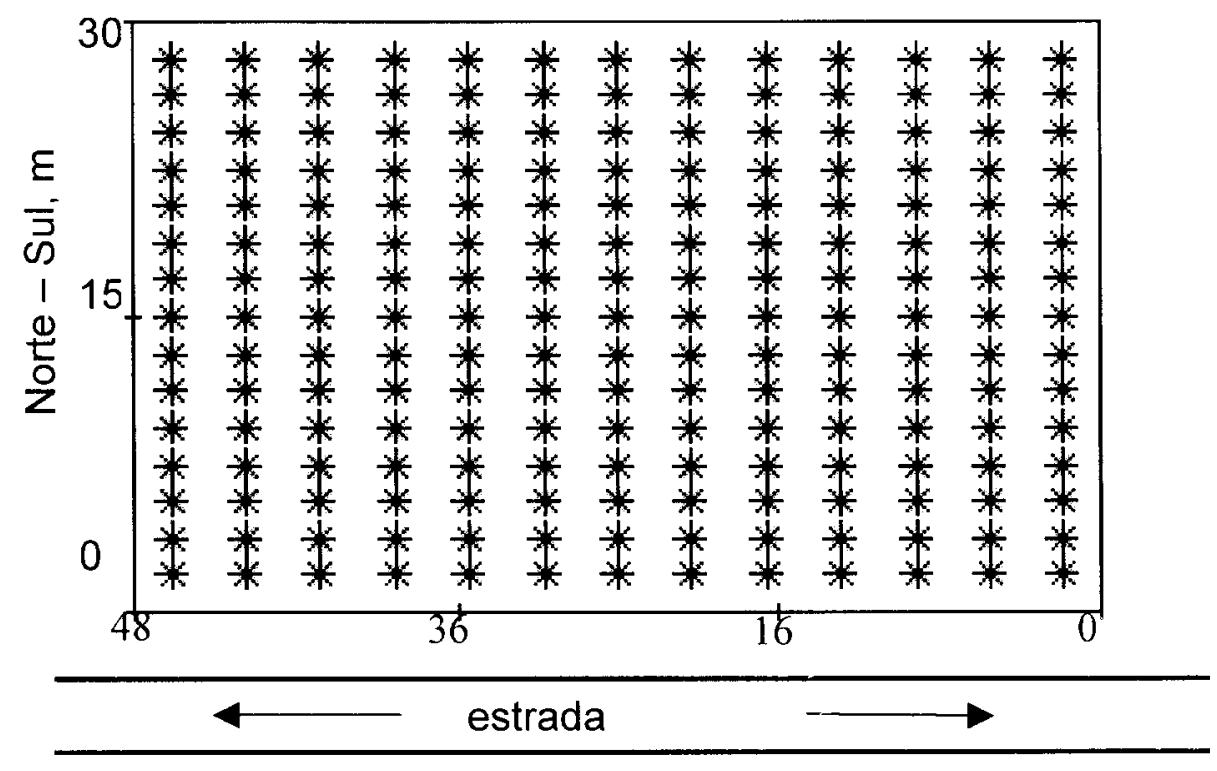

Leste - Oeste, $\mathrm{m}$

Figura 1 - Croqui dos pontos amostrados das áreas no Argissolo Vermelho-Amarelo em Santa Maria (a), no Latossolo Vermelho distrófico em Cruz Alta, RS e no Latossolo Vermelho distroférrico típico, em Coronel Barros (b). Asteriscos indicam os pontos amostrados.

metros até próximo dos 24 metros para o interior da lavoura e o menor estado de compactação foi entre 24 metros e 36 metros aproximadamente, com RP variando entre 0,58 a $0,88 \mathrm{MPa}$.

Para o Latossolo Vermelho distrófico, os mapas de superfície indicaram que o maior estado de compactação variou de 2,76 até 3,20MPa (Figura 2b). O intermediário estado de compactação variou de 2,23 até $2,75 \mathrm{MPa}$, e o menor estado de compactação foi de 1,70 até $2,22 \mathrm{MPa}$.

Assim como no Argissolo, o Latossolo Vermelho distroférrico típico apresentou uma distribuição da RP em faixas, saindo de um estado de maior compactação na cabeceira da lavoura e diminuindo para o centro da lavoura (Figura 2c). O maior estado de compactação apresentou valores de RP variando de 2,11 a 2,80MPa, para o intermediário estado de compactação considerou-se a RP variando de 1,60 a 2,10MPa e o menor estado de compactação, 0,20 a 1,59MPa.

De acordo com os resultados dos mapas de RP, estimados pela krigagem, aceita-se a hipótese que o tráfego de máquinas, principalmente em lavouras sob plantio direto, provoca diferentes estados de compactação do solo e que o maior estado de compactação ocorre na extremidade da lavoura.

\section{CONCLUSÕES}

A normalidade dos valores de resistência à penetração é variável para tipos de solos e 


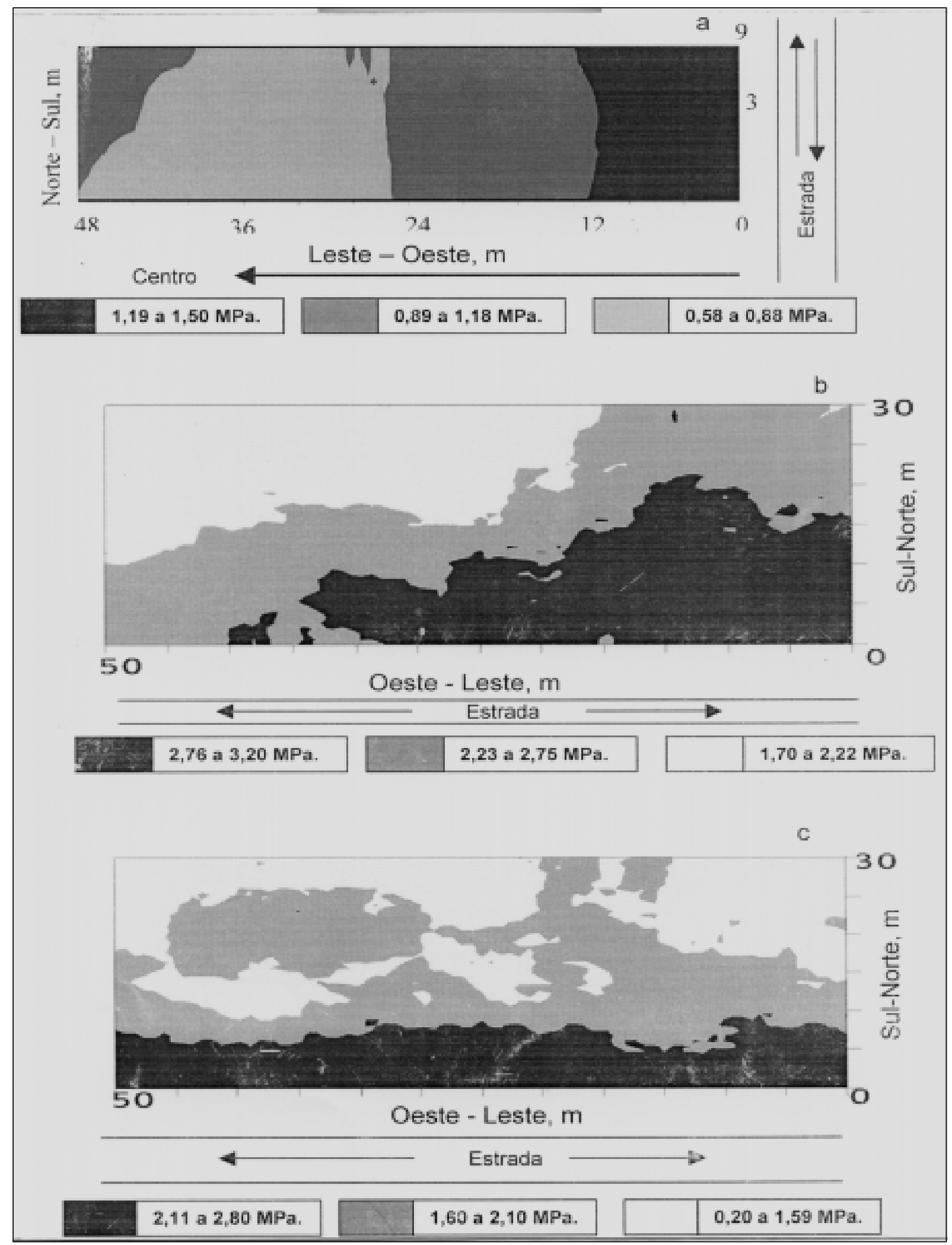

Figura 2 - Superfície de resistência do solo à penetração na profundidade de 7,5 cm no Argissolo Vermelho-Amarelo distrófico (a), no Latossolo Vermelho distrófico (b) e no Latossolo Vermelho distroférrico típico (c), identificando os estados de compactação. 
profundidades, geralmente, nas camadas superficiais e aquelas que recebem o efeito do tráfego de máquinas apresentam distribuição aleatória, diferente da normal.

Nos semivariogramas que apresentaram estacionaridade definida, o alcance foi de aproximadamente 3 metros no Argissolo VermelhoAmarelo, 13,6 metros no Latossolo Vermelho distrófico e 17 metros no Latossolo Vermelho distroférrico típico.

Em lavouras sob plantio direto, o tráfego de máquinas provoca distintos estados de compactação. O maior estado de compactação ocorre na região dita "cabeceira" da lavoura, onde as máquinas realizam as manobras e o estado de compactação vai reduzindo-se para o centro da lavoura.

\section{REFERÊNCIAS BIBLIOGRÁFICAS}

ABREU, L.S. Propriedades hídricas e mecânicas afetadas por sistemas de manejo e variabilidade espacial de um Argissolo. 2000. 66f. Dissertação (Mestrado em Agronomia) - Programa de Pós-graduação em Agronomia, Universidade Federal de Santa Maria.

CAMBARDELLA, C.A. et al. Field scale variability of soil properties in Central Iowa soils. Soil Science Society of America Journal, v.58, p.1501-1511, 1994.

DARIVA, T.A. Variabilidade espacial de solo e da cultura da soja em várzea sistematizada. 2001. 120f. Dissertação (Mestrado em Agronomia) - Programa de Pós-graduação em Agronomia, Universidade Federal de Santa Maria.

EMPRESA BRASILEIRA DE PESQUISA AGROPECUÁRIAEMBRAPA. Centro Nacional de Pesquisa de Solos. Sistema brasileiro de classificação de solos. Rio de Janeiro, 1999. $412 \mathrm{p}$.

GAMMA DESIGN SOFTWARE. Geoestatistics for the environmental sciences (version 5.1 for windows). Michigan : 2000. $1 \mathrm{CD}$.

GONÇALVES, A.C.A.; FOLEGATTI, M.V.; SILVA, A.P. Estabilidade temporal da distribuição espacial da umidade do solo em área irrigada por pivô central. Revista Brasileira de Ciência do Solo, v.23, p.155-164, 1999.
HAKANSSON, I.; MEDVEDEV, V.W. Protection of soils from mechanical overloading by establishing limits for stresses caused by heavy vehicles. Soil \& Tillage Research, v.35, p.85-97, 1995 .

KERTZMANN, F.F. Modificações na estrutura e no comportamento de um Latossolo Roxo provocados pela compactação. 1996. 153f. Tese (Doutorado em Geografia Física) - Faculdade de Filosofia, Letras e Ciências Humanas, Universidade de São Paulo.

KLEIN, V.A. Propriedades físico-hídrico-mecânicas de um Latossolo Roxo, sob diferentes sistemas de uso e manejo. 1998. 130f. Tese (Doutorado em Agronomia) Universidade de São Paulo, Escola Superior de Agricultura Luiz de Queiroz.

SILVA, M.L.N.; CURI, N.; BLANCANEAUX, P. Sistemas de manejo e qualidade estrutural de Latossolo Roxo. Pesquisa Agropecuária Brasileira, v.35, p.2485-2492, 2000a.

SILVA, V.R.; REINERT, D.J.; REICHERT, J.M. Resistência mecânica do solo à penetração influenciada pelo tráfego de uma colhedora em dois sistemas de manejo do solo. Ciência Rural, v.30, p.795-801, 2000b.

SNEDECOR, G.W.; COCHRAN, W.G. Statistical methods. 7.ed. Ames : Iowa State University, 1974. 507p.

SOUZA, L.S.; COGO, N.P.; VIEIRA, S.R. Variabilidade de propriedades físicas e químicas do solo em um pomar cítrico. Revista Brasileira de Ciência do Solo, v.21, p.367-372, 1997.

SOUZA, Z.M. et al. Variabilidade espacial de atributos físicos em um Latossolo Vermelho Distrófico sob semeadura direta, em Selvíria, MS. Revista Brasileira de Ciência do Solo, v.25, p.699-707, 2001.

UTSET, A.; CID, G. Soil penetrometer resistance spatial variability in a Ferrosol at several soil moisture conditions. Soil \& Tillage Research, v.61, p.193-202, 2001.

VOORHEES, W.B.; SENST, C.G.; NELSON, W.W. Compaction and soil structure modification by wheel traffic in the northern Corn Belt. Soil Science Society of America Journal, v.42p.344-349, 1978.

WARRICK, A.W.; NIELSEN, D.R. Spatial variability of soil physical properties in the field. In: HILLEL, D. (ed.). Applications of soil physics. New York : Academic, 1980. p.319-376. 